\title{
Dynamics of optically levitated microparticles in vacuum placed in 2D and 3D optical potentials possessing orbital angular momentum
}

Yoshihiko Arita, Michael Mazilu, Mingzhou Chen, Tom Vettenburg, Juan M. Auñón, et al.

Yoshihiko Arita, Michael Mazilu, Mingzhou Chen, Tom Vettenburg, Juan M. Auñón, Ewan M. Wright, Kishan Dholakia, "Dynamics of optically levitated microparticles in vacuum placed in 2D and 3D optical potentials possessing orbital angular momentum," Proc. SPIE 10252, Optical Manipulation Conference, 102520V (18 April 2017); doi: 10.1117/12.2275309

Event: SPIE Technologies and Applications of Structured Light, 2017, Yokohama, Japan 


\title{
Dynamics of optically levitated microparticles in vacuum placed in $2 \mathrm{D}$ and $3 \mathrm{D}$ optical potentials possessing orbital angular momentum
}

\author{
Yoshihiko Arita, ${ }^{1,2, *}$ Michael Mazilu, ${ }^{1}$ Mingzhou Chen, ${ }^{1}$ Tom Vettenburg, ${ }^{1}$ Juan M. \\ Auñón, ${ }^{1}$ Ewan M. Wright ${ }^{1,3}$ and Kishan Dholakiaa ${ }^{1,3, *}$ \\ ${ }^{1}$ SUPA, School of Physics \& Astronomy, University of St Andrews, North Haugh, St Andrews, Fife, KY16 9SS, UK \\ ${ }^{2}$ Molecular Chirality Research Centre, Graduate School of Advanced Integration Science, Chiba University, 1-33 \\ Yayoi, Inage, Chiba, 263-0022, Japan \\ ${ }^{3}$ College of Optical Sciences, The University of Arizona, 1630 East University Boulevard, Tucson, Arizona \\ 85721-0094, USA \\ *ya10@st-andrews.ac.uk or kd1@ @st-andrews.ac.uk
}

\begin{abstract}
We demonstrate the transfer of orbital angular momentum to optically levitated microparticles in vacuum [1]. We prepare two-dimensional and three-dimensional optical potentials. In the former case the microparticle is placed within a Laguerre-Gaussian beam and orbits the annular beam profile with increasing angular velocity as the air drag coefficient is reduced. We explore the particle dynamics as a function of the topological charge of the levitating beam. Our results reveal that there is a fundamental limit to the orbital angular momentum that may be transferred to a trapped particle, dependent upon the beam parameters and inertial forces present. This effect was predicted theoretically [2] and can be understood considering the underlying dynamics arising from the link between the magnitude of the azimuthal index and the beam radius [3].

Whilst a Laguerre-Gaussian beam scales in size with azimuthal index $\ell$, recently we have created a "perfect" vortex beam whose radial intensity profile and radius are both independent of topological charge $[4,5]$. As the Fourier transform of a perfect vortex yields a Bessel beam. Imaging a perfect vortex, with its subsequent propagation thus realises a complex three dimensional optical field. In this scenario we load individual silica microparticles into this field and observe their trajectories. The optical gradient and scattering forces interplay with the inertial and gravitational forces acting on the trapped particle, including the rotational degrees of freedom. As a result the trapped microparticle exhibits a complex three dimensional motion that includes a periodic orbital motion between the Bessel and the perfect vortex beam. We are able to determine the three dimensional optical potential in situ by tracking the particle. This first demonstration of trapping microparticles within a complex three dimensional optical potential in vacuum opens up new possibilities for fundamental studies of many-body dynamics, mesoscopic entanglement $[6,7]$, and optical binding $[8,9]$.
\end{abstract}

OCIS codes: Levitated optomechanics, Orbital angular momentum, Laguerre-Gaussian beam, Perfect vortex

\section{References}

1. M. Mazilu, Y. Arita, T. Vettenburg, J. M. Auñón, E. M. Wright, and K. Dholakia, "Orbital-angularmomentum transfer to optically levitated microparticles in vacuum," Phys. Rev. A 94, 053821 (2016).

2. J. Ng, Z. F. Lin, and C. T. Chan, "Theory of optical trapping by an optical vortex beam," Phys. Rev. Lett. 104, 103601 (2010).

3. M. J. Padgett, F. M. Miatto, M. P. J. Lavery, A. Zeilinger, and R. W. Boyd, "Divergence of an orbital-angularmomentum-carrying beam upon propagation,” New J. Phys. 17, 023011 (2015).

Optical Manipulation Conference, edited by Takashige Omatsu, Ryuji Morita, Proc. of SPIE Vol.

10252, 102520V · (c) 2017 SPIE · CCC code: 0277-786X/17/\$18 · doi: 10.1117/12.2275309 
4. M. Chen, M. Mazilu, Y. Arita, E. M. Wright, and K. Dholakia, "Dynamics of microparticles trapped in a perfect vortex beam," Opt. Lett. 38, 4919 (2013).

5. M. Chen, M. Mazilu, Y. Arita, E. M. Wright, and K. Dholakia, "Creating and probing of a perfect vortex in situ with an optically trapped particle," Opt. Rev. 22, 162 (2015).

6. D. E. Chang, C. A. Regal, S. B. Papp, D. J. Wilson, J. Ye, O. Painter, H. J. Kimble, and P. Zoller, "Cavity opto-mechanics using an optically levitated nanosphere," Proc. Natl. Acad. Sci. USA 107, 1005 (2010).

7. O. Romero-Isart, M. L. Juan, R. Quidant, and J. I. Cirac, "Toward quantum superposition of living organisms," New J. Phys. 12, 033015 (2010).

8. Y. Arita, M. Mazilu, T. Vettenburg, E. M. Wright, and K. Dholakia, "Rotation of two trapped microparticles in vacuum: observation of optically mediated parametric resonances," Opt. Lett. 40, 4751 (2015).

9. K. Dholakia and P. Zemánek, "Colloquium: Gripped by light: Optical binding," Rev. Mod. Phys. 82, 1767 (2010). 